\title{
A testing technique to investigate the tensile behavior of propellant representative material
}

\author{
Thibaut Viant ${ }^{1,2}$, Pascal Forquin ${ }^{1 *}$, Dominique Saletti ${ }^{1}$, Didier Imbault ${ }^{1}$, Pierre Brunet $^{2}$, Julien Moriceau ${ }^{2}$, Gilles Poirey $^{2}$ \\ ${ }^{1}$ Univ. Grenoble Alpes, 3SR, F-38000 Grenoble, France \\ ${ }^{2}$ Ariane Group, "le Bouchet" Research Center, F-91710 Vert-Le-Petit, France
}

\begin{abstract}
Propellants are energetic materials abundantly used to generate the propulsion of rockets, projectiles, or other objects. A wide range of stress-state and strain-rate has to be considered in view of predicting the mechanical behaviour of this material over its different life cycles. Propellant materials are usually studied through the use of propellant representative materials (PRM) when studied in a university laboratory. In the present work an experimental device was developed to investigate the dynamic tensile response of a PRM material. This device is based on the use of a pendulum that is employed to dynamically load the PRM sample on one end. The sample is attached to an instrumented Hopkinson bar on the other end. The data processing of Hopkinson bar point measurements combined to DIC (digital-imagecorrelation) measurements allows the stress and strain levels in the sample to be characterised. Finally these experimental results can be used to enhance the constitutive modelling of PRM materials.
\end{abstract}

\section{Introduction}

Propellants are energetic materials abundantly used to generate the propulsion of rockets, projectiles, or other objects. A wide range of stress-state and strain-rate has to be considered in view of predicting the mechanical behaviour of this material over its different life cycles. Propellant materials are usually studied through the use of propellant representative materials (PRM) when studied in a university laboratory.

The aim of studying this representative material is to fully understand the mechanical behaviour of energetic materials and an experimental approach can be adopted in order to improve the different modelling behaviour used to predict the material response to different life cycles.

The tensile behaviour of PRM under quasi-static and intermediate strain rates (up to $100 / \mathrm{s}$ ) is important to be properly determined in order to determine the microcrack states that can result from an accidental event, such as a mishandling or a fall of a tank containing the material.

PRM is a viscoelastic material made of a polymeric matrix and 5-to-200 $\mu \mathrm{m}$ mean diameter particles replacing the active charges present in the real propellant, for sake of safety. Then, strain-rate sensitivity is expected and has to be fully investigated.

This paper is a part of the whole study focusing on the study of the tensile behaviour of PRM at intermediate strain-rates. To do so, a dedicated experimental set-up has been developed in 3SR laboratory as described in the next section. Then, some results will be presented and perspectives will be drawn in a final section.

\section{Experimental technique}

As previously mentioned, the experimental set-up used for this study was especially developed to characterise the mechanical response of PRM under intermediate tensile loading. It composed of two main parts and depicted in the Fig. 1.

The first part is dedicated to the application of the dynamic tensile loading thanks to a hammer-shaped double pendulum system. This pendulum hits one part attached to one end of the specimen. The displacement history is obtained via a laser interferometer technique.

The second part of the setup is a $6 \mathrm{~m}$ long slender bar used as a Hopkinson output bar measurement. The specimen transmits its loading to this bar which is instrumented with three strain gauges and tied to the ground at its end. This bar allows measuring the force history applied on the specimen during the test. The gauge signals are post-processed with the Emilie software [1].

A painted speckle is applied on the surface of the specimen (see the figure 2) in order to capture the whole test sequence with a fast camera. The fame rate acquisition is set to $15 \mathrm{kfps}$ and the observation window is 640 by 480 pixels. The sequence is processed with the Correli-LMT digital image correlation algorithm [2] adapted to dynamic tensile test [3]. 


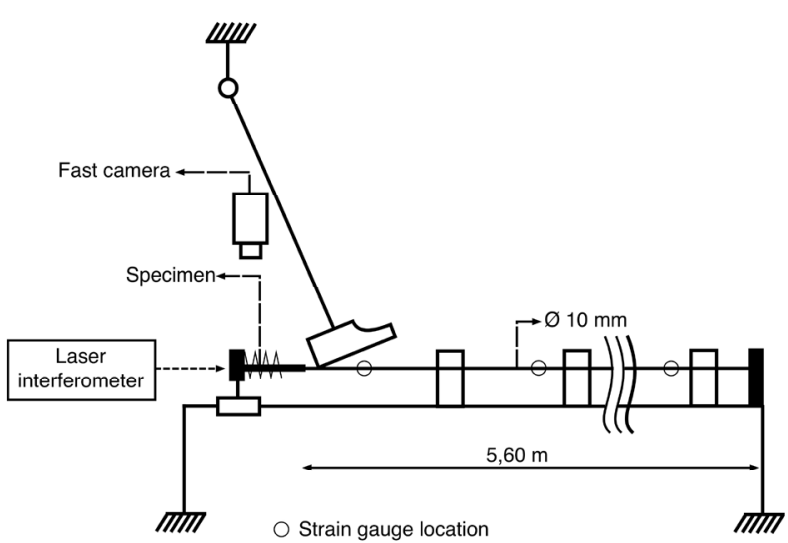

Fig. 1. Sketch of the experimental setup developed to test PRM (Propellant Representative Materials) and other soft materials (ExperDYN platform, 3SR lab.) [4].

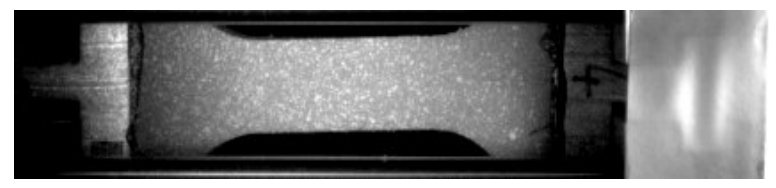

Fig. 2. View of the tested sample. A painted speckle is applied.

Finally, in order to obtain an approximately constant strain rate, springs have been interposed between the hammers and the mobile jaw as shown in Fig. 3. Numerical simulations have been done to optimize the length and the stiffness of springs.

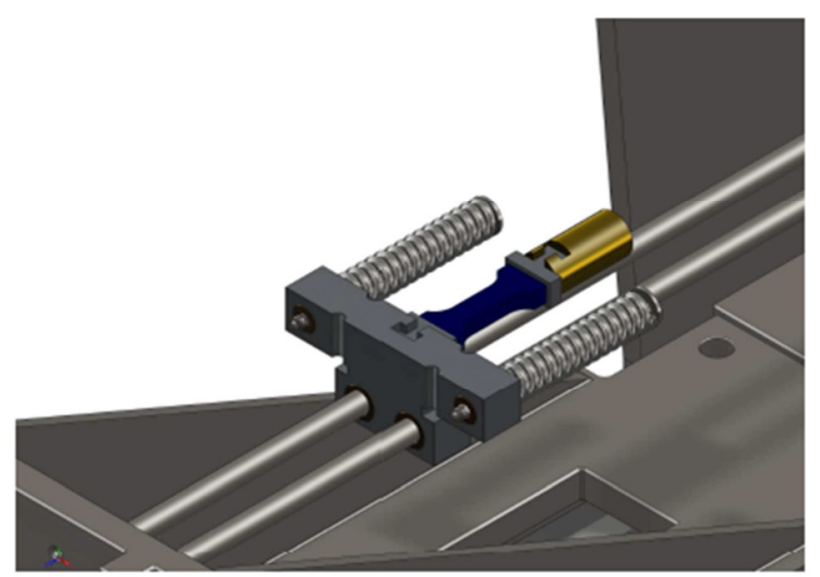

Fig. 3. Detailed view of the tensile device (CAD Software).

\section{Experimental results}

Since forces and velocities are given by the Emilie software, a signal transportation can be handled to find the stress and strain undergone by the specimen. To obtain further information, DIC analyses are performed. The figure 4 shows a corrected stress-strain curve. One can observe that the strain-rate is close to be constant during the test and equals to $100 \mathrm{~s}^{-1}$. Moreover, the stress rises linearly up to $2 \mathrm{MPa}$ and seems to reach a yield stress at about 3.5 MPa.

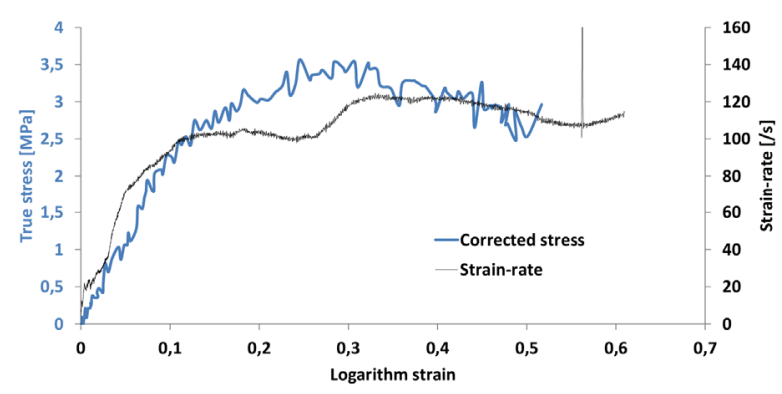

Fig. 4. Experimental result of a dynamic tensile test performed with a PRM sample. Corrected stress-strain curve and associated strain-rate.

\section{Conclusion}

This paper deals with a new technique to investigate the dynamic behaviour of ductile materials. The setup has been designed to achieve a tensile test with a long duration time (up to $10 \mathrm{~ms}$ ) at an applied strain-rate which must be constant as possible.

By the use of Correli-LMT software, a corrected true stress can be calculated and drawn versus the logarithm strain. This experimental setup has allowed discovering the behaviour of the PRM at a strain-rate of $100 \mathrm{~s}^{-1}$. By releasing hammers from different heights, a [50-200 $\left.\mathrm{s}^{-1}\right]$ strain-rate range can be explored. The obtained experimental results lead to a better understanding and modelling of the tensile behaviour of PRM and propellant materials at elevated strain-rates.

This setup has been designed to characterize filled elastomers but can also be used to investigate dynamic tensile behaviour of other materials by adapting hammers mass and fall heights.

\section{References}

1. Bussac M.-N., Collet, P., Gary, G., Othman, R., Journal of the Mechanics and Physics of Solids 50, 321-349 (2002)

2. Besnard, G., Hild, F., Roux, S., Applied optics, Experimental Mechanics 46:6, 789-804. (2006)

3. Saletti, D., Pattofatto, S., Zhao, H., Mechanics of Materials 65, 1-11 (2013)

4. Forquin, P., The ExperDYN dynamic testing platform, http://www.3srgrenoble.fr/sites/3srgrenoble.fr/IMG/pdf/its/ExperD yn.pdf 